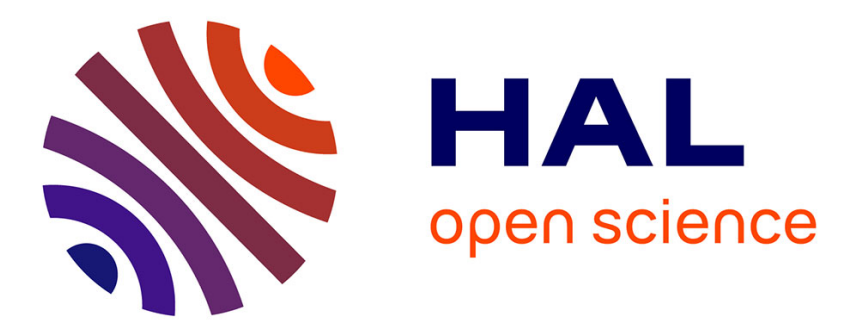

\title{
A collaborative decision-making approach for supply chain based on a multi-agent system
}

\author{
Yacine Ouzrout, Abdelaziz Bouras, El Habib Nfaoui, Omar El Beqqali
}

\section{To cite this version:}

Yacine Ouzrout, Abdelaziz Bouras, El Habib Nfaoui, Omar El Beqqali. A collaborative decisionmaking approach for supply chain based on a multi-agent system. Benyoucef, Lyes; Grabot, Bernard (Eds.). Artificial Intelligence Techniques for Networked Manufacturing Enterprises Management, Springer, pp.520, 2010, Springer Series in Advanced Manufacturing. hal-00442392

\section{HAL Id: hal-00442392 \\ https://hal.science/hal-00442392}

Submitted on 30 Oct 2018

HAL is a multi-disciplinary open access archive for the deposit and dissemination of scientific research documents, whether they are published or not. The documents may come from teaching and research institutions in France or abroad, or from public or private research centers.
L'archive ouverte pluridisciplinaire HAL, est destinée au dépôt et à la diffusion de documents scientifiques de niveau recherche, publiés ou non, émanant des établissements d'enseignement et de recherche français ou étrangers, des laboratoires publics ou privés. 


\title{
A collaborative decision-making approach for supply chain based on a multi-agent system
}

\author{
Yacine Ouzrout', Abdelaziz Bouras' ${ }^{1}$ El Habib Nfaoui ${ }^{12}$, Omar El Beqqali², \\ ${ }^{1}$ LIESP Laboratory - Université de Lyon - Université Lumière Lyon 2 \\ 160 Bd de l'université 69676 Bron, France \\ ${ }^{2}$ GRMS2I - FSDM/ Université Sidi Md Ben Abdellah, \\ B.P 1796 Fès-Atlas, Morocco \\ \{yacine.ouzrout, abdelaziz.bouras, elhabib.nfaoui\}@univ-lyon2.fr, obekkali@fsdmfes.ac.ma
}

\begin{abstract}
To improve the supply chain's performance under demand uncertainty and exceptions, various levels of collaboration techniques based on information sharing were set up in real supply chains (VMI, CPR, CPFR...). The main principle of these methods is that the retailers do not need to place orders because wholesalers use information centralization to decide when to replenish them. Although these techniques could be extended to a whole supply chain, current implementations only work between two business partners. With these techniques, companies electronically exchange a series of written comments and supporting data, which includes past sales trends, scheduled promotions, and forecasts. This allows participants to coordinate joint forecasting by focusing on differences in forecasts. But if the supply chain consists of autonomous enterprises, sharing information becomes a critical obstacle, since each independent actor is typically not willing to share with the other nodes its own strategic data (as inventory levels); That is why researchers proposed different methods and information systems to let the members of the supply chain collaborate without sharing all their confidential data and information. In this chapter we analyze some of the existing approaches and works and describe an agent-based distributed architecture for the decision-making process. The agents in this architecture use a set of negotiation protocols (such as Firm Heuristic, Recursive Heuristic, CPFR Negotiation Protocol) to collectively make decisions in a short time. The architecture has been validated on an industrial case study.
\end{abstract}

Keywords: Multi-Agent Systems, Supply Chain Management, Decision-making, Distributed Simulation. 


\section{Introduction}

The economic and industrial communities worldwide are confronted with the increasing impact of competitive pressures resulting from the globalization of markets and supply chains (SC) for product fulfillment. More and more companies are being driven to pursue new forms of collaboration and partnership with their direct logistics counterparts. As a result, at a company level there is a progressive shift towards an external perspective with the design and implementation of new management strategies, which are generally named with the term of supply chain management (SCM). However, in order to conduct this concept in practice, several hurdles are still to overcome (Samii 2004) (Hieber 2002), mainly due to:

The conflicts resulting from local objectives versus network strategies, because supply chain is a multi decisional context, so companies must make decisions collectively.

- The difficulty in making decisions in a collaborative manner. It can be observed in several supply chain cases.

The need for sharing sensitive information of participants in the SC. If the supply chain is composed by independent companies, sharing information becomes a critical obstacle; since each independent actor is typically not willing to share with the other nodes its own strategic data (as production capacity, internal lead times, production costs, sales forecasts, etc.).

- $\quad$ The need for sharing information technology tools.

Supply Chain Management (SCM) has demanded attention and support from the industrial community. It consists in the coordination of production, inventory, location, and transportation among the participants in a supply chain to achieve the best mix of responsiveness and efficiency for the market being served (Hugos, 2003a). The optimal deployment of inventory is one of the main goals of SCM. Indeed, Many collaborative processes (e.g. CPFR: Collaborative Planning, Forecasting and Replenishment (VICS Association 2007), VMI: Vendor Managed Inventory (John Taras CPIM 2007), CRP: Continuous Replenishment Program and ECR: Efficient Consumer Response (ECR 2006) and software systems (e.g. APS: Advanced Planning Systems (Simchi-Levi et al. 2000), ERP: Enterprise Resource Planning (Baglin et al. 2001) are used for management and control of inventory in order to reduce the total system cost of inventory as much as possible while still maintaining the service levels that customers require. Literature shows that the common objectives of these practices is to avoid the surplus inventory, reduce the inventory shortage, minimize the safety stock, produce and deliver products in the right quantities and at the right time. However for the distributor centers, it is difficult to achieve this goal, because the rush unexpected orders placed by the wholesalers always present a challenge. This challenge will vary from one company to another and from one supply chain to another. In fact, the distributor can not predict the date and the ordered quantity of this type of orders since it is a random one whose causes is multiple and depends closely on the branch of industry concerned. In addition, this type of orders has a very short delivery date. In this emergency case, the distributor is 
not able to wait for the next planned delivery of products from the supplier. Therefore, generally the order can be cancelled or can cause an inventory shortage if the ordered quantity is large. This will have a bad impact on the quality of the offered service within the satisfaction of the final customer policy.

Some suppliers allocate an additional human resources and logistics for delivering the rush unexpected orders of their distributors. The disadvantage of this solution is that the costs suggested are generally very high.

In multi-echelon networks, which is a common distribution model for many distributors and manufacturers, the distributors can deliver the rush unexpected orders. The echelon inventory includes the sum of local stock and the stock of all the forward distribution centers (Calvin 2003) (Siala et al. 2006). However, multi-echelon inventory management is more consistent with the centralized decisions. It requires that all locations must be submitted to the relevant control of a single company. In addition, it requires a high degree of information sharing between the various actors of the SC, but if the supply chain consists in independent companies, information sharing becomes a critical obstacle, since each independent actor is typically not willing to share with the other nodes its own strategic data (as inventory levels). Also, it monitors his inventory levels (by using autonomous action and policies) and places the orders to its suppliers in order to optimize its own objective (Siala et al. 2006).

This chapter focuses on unexpected swings in demand and on unexpected exceptions (problem of production, problem of transportation, etc.), which are important coordination and communication issues in SC management (Giannoccaro et al. 2003) (Reutterer and Kotzab 2000) (Zhao et al. 2002). Both events can engender the presence of a rush unexpected orders in a node of supply chain; in particular, at the wholesalers and the distribution center levels. In this context, we propose a collaborative process which presents an effective solution (to the distributors) for better management of the rush unexpected orders for which the quantity of product cannot be delivered partially or completely from the available inventory. This process includes the distributors of the same or equivalent products and their wholesalers. The participants in the process can be competitors. To implement the process, we apply an agent-based distributed architecture in order to guarantee the autonomy and the strategic data confidentiality of all participants. Agent technology provides to the distributed environment a great promise of effective communication (Swaminathan et al. 1998). An agent is a program that performs a specific task intelligently without any human supervision and can cooperatively communicate with other agents. Therefore, agent technology is suitable to solve communication concerns for a distributed environment. Recent researches also show that the multi-agent approach plays a significant role in supply chain management, for example (Wu 2001), (Fu et al. 2000), (Kimbrough et al. 2002) and (Swaminathan et al. 1998).

This chapter is structured as follows. In Section 2 we introduce the role of distributed simulation in the supply chain context. In Section 3 we show a generic model fixing and defining the supply chain elements to be considered and the modeling methodology. In sections 4 and 5 we propose a distributed decision-making 
architecture based on a multi-agent system and we implement this system on a real industrial case study. Finally, we conclude the chapter and give an overview of the research perspectives.

\section{Distributed simulation and supply chain management}

Many software vendors (e.g. IBM in (Bagchi et al. 1998)), universities and companies (Telle et al. 2003) (Banks et al. 2002) have traditionally used a local simulation approach in the supply chain context. Only in recent years, more and more companies in supply chain adopt distributed collaborative simulation (Brun et al. 2002), because it provides a connection between supply chain nodes that are geographically distributed throughout the globe, guaranteeing that each single simulation model is really linked to its respective industrial site. Moreover, companies in any supply chain must make decisions individually and collectively regarding their actions in production, inventory, location, transportation and information, then the distributed simulation can preserve at the same time the local autonomies and privacy of logistics data. In some cases, the execution of a distributed model allows to reduce the time spent for simulation, since separated models run faster than a single complex model (Fujimoto et al. 1999).

Despite the great use of simulation in SC and SCM, there are many additional opportunities for application of the methodology (Banks et al. 2002). However, many of these opportunities require that challenges be overcome (see Introduction). Aim of the agent-based distributed simulation presented in this chapter is:

To convince decision-makers to adopt a SCM process and to choose the most appropriate management strategies and practices for a given SC.

To make decisions collectively in a short time. Mainly, in the case of operational planning (short term, for example: rush order) (Pinedo and Chao 1999) or in a situation where the supply chain partners negotiate a delivery date modification due to a disturbance (for example: problem of production, problem of transport, etc.), because the decision system has to make its choice within a short time, and must be able to evaluate the consequences regarding various scenario in distributed manner within a shorter time too.

\subsection{Decision-making and multi-agent approaches}

In order to have a flexible and proactive model, we have chosen the Multi-Agent approach to develop our architecture. Clautier (Clautier et al. 2001) and Parunak (Parunak 1996) showed the main benefit from using this approach in the field of the supply chain. Thus, the complete architecture of simulation is made on a set of agents modeling the supply chain participants. These collaborative agents communicate between them and negotiate using protocols. They seek the accurate and timely data that hold the promise of better coordination and better decision-making in the information systems of the supply chain participants (such as an ERP (Enterprise 
Resources Planning) system); this means every time the simulation starts, the model must be initialized with the current states of the supply chain participants.

In the literature, various researches have been proposed to compensate for the uncertainty that exists in a supply chain. Cohen and Lee (Cohen and Lee 1988) have developed a planning model to optimize material supply, production and distribution processes. Arntzen et al. (Arntzen et al. 1995) have proposed a resource allocation and planning model for global production and distribution networks. Kimbrough and Zhong (Kimbrough and Zhong 2002), McBurney et al. (McBurney et al. 2002), Chen et al. (Chen et al. 2000a; 2000b) focused on demand forecasting. Most of these researches suppose that companies in the supply chain share the information and coordinate the orders. But if the supply chain consists of autonomous companies, sharing information becomes a critical obstacle, since each independent actor is typically not willing to share with the other nodes its own strategic data (as inventory levels) (Terzi and Cavalieri 2004); An example is the case of several competitor wholesalers (located in the same or different geographical areas) which source of the same distributor.

\subsection{Multi-Agent Systems Simulation}

Various projects applied the multi-agent system paradigm to solve different problems in SC (as inventory planning, demand and sales planning, distribution and transportation planning, etc). DragonChain was implemented by Kimbrough and Zhong (Kimbrough and Zhong 2002) at the University of Pennsylvania (Philadelphia, PA, USA) to simulate supply chain management, and more particularly to reduce bullwhip effect. For that, they base their simulation on two versions of the Beer Game, the MIT Beer Game (i.e. the original game) and the Columbia Beer Game, and they use agents that look for the best ordering scheme with genetic algorithms. Maturana et al. (Maturana et al. 1999) have developed a hybrid agent-based mediatorcentric architecture, called MetaMorph, to integrate partners, suppliers and customers dynamically with the main company through their respective mediators within a supply chain network via the Internet and Intranets. In MetaMorph, agents can be used for representing manufacturing resources (machines, tools, etc.) and parts, encapsulating existing software systems and functioning as system or subsystem coordinators/mediators. Swaminathan et al. (Swaminathan et al. 1998) have proposed a multi-agent approach to model supply chain dynamics. In their approach, a supply chain library of software components, such as retailers, manufacturers, inventory policy, and so on, has been developed to build customized supply chain models from the library. Sadeh et al. (Sadeh et al. 2001) have developed an agent-based architecture for a dynamic supply chain called MASCOT. The MASCOT is a reconfigurable, multilevel, agent-based architecture for a coordinated supply chain. Agents in MASCOT serve as wrappers for planning and scheduling modules. Petersen et al. (Petersen et al. 2001) have proposed a multi-agent architecture, called AGORA, for modeling and supporting cooperative work among distributed entities in virtual enterprises. Nfaoui et al. (Nfaoui et al., 2006) also proposed an agent-based distributed architecture for simulation in decision-making process within the supply 
chain context. Agents in this architecture use a set of negotiation protocols (such as Firm Heuristic Negotiation, Recursive Heuristic Negotiation, CPFR Negotiation Protocol) to make decisions collectively in a short time. Chehbi et al. (Chehbi et al. 2003) have proposed a multi-agent supply chain architecture to optimize distributed decision making, and finally Moyaux et al. (Moyaux et al. 2004) have developed an agent simulation model for the Québec forest supply chain.

\section{The supply chain modeling}

Two parameters are important in the process of the modeling of a supply chain, the perimeter and the structure. The first delimits the supply chain in terms of number of actors (companies), and the second defines the customer/supplier relationships. If the two aforementioned parameters are not described, it is difficult to define the modeling boundaries (in term of levels). Indeed, a supply chain can use several tens, even hundreds of nodes geographically distributed throughout the globe. Is it really necessary to take into account all the actors? Moreover, a company can belong to several supply chains. Then, what are the levels of customers and suppliers that should be covered by the proposed model?

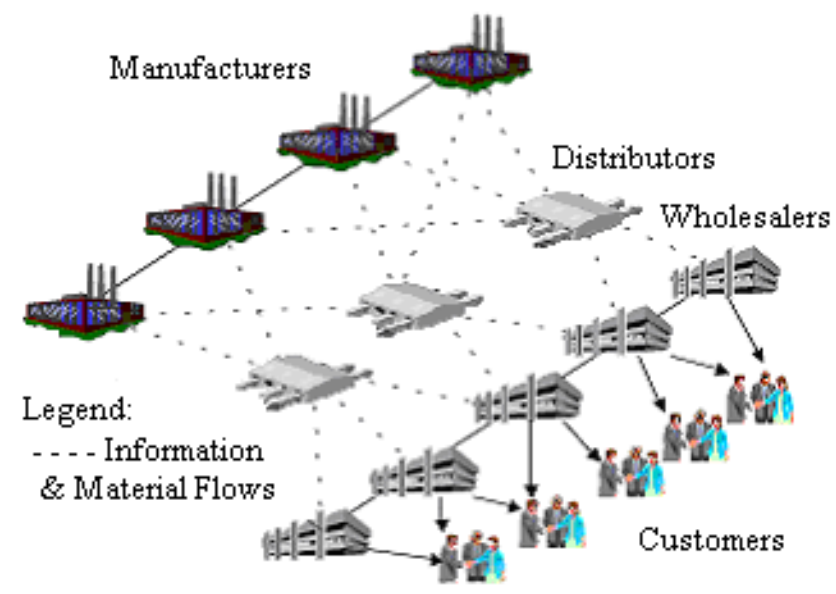

Fig1. Overview of a global distribution supply chain

\subsection{SC modeling methodology}

To answer these two questions, it is necessary to identify the product for which the supply chain is defined. Indeed, we define a supply chain for a product or a family of products. It is composed of all the companies involved in the design, production, 
transportation, and delivery of a product to market. Having a clear knowledge of the product, we better specify the central company of the supply chain, i.e. the one that assembles the finished product. Next, we propose to follow the steps below, they define a generic model for supply chain which delimits the boundaries of modeling and defines the customers/suppliers relationships:

1. Identify the finished product for which the supply chain is defined. This automatically defines the core company (central company) of the supply chain.

2. Identify the bill of materials of the finished product.

3. Exclude from this bill of materials the raw materials not requiring a partnership or collaboration.

4. Afterwards, identify the remaining raw materials suppliers (distributors or factories having the activity of production). The same raw material can be bought from one or more different suppliers. In this last case, the percentage of the orders to place to each one of them should be determined.

5. For each supplier, the steps 2,3 and 4 have to be renewed by considering, this time, the raw material as a finished product, and so on up to the upstream supplier.

6. Determine the type of the orders (stationary, random, etc.) of the customers (other than central company) of the various suppliers identified in step 4.

7. Remake step 6 for the suppliers of the suppliers except for the last suppliers (upstream suppliers).

8. Identify the list of customers of central company.

9. In this list, identify the potential customers acting on the supply chain of the product (customers requiring collaboration or a partnership) and those which do not require the collaboration. Then, determine the type of the orders for the latter (random, stationary, etc.) as well as the percentage of the orders which each potential customer places to central company.

10. For each potential customer of central company, remake steps 8 and 9 except for the final customers (for example, consumers).

Agents-based Model:

11. Allot an Agent-based model to all the identified actors (central company, customers and suppliers).

To represent the three main functions of the company (source, make and deliver) and consider the control processes in the supply chain and its environment, each actor is modeled by seven agents. These agents are: AgentPRC which plays the part of the processes related to the customer, AgentDis which manages the "distribution storage", AgentPro which plays the role of the "make" process, AgentApp for the "source" process, AgentAch which plays the role of the "purchase" process, AgentSCM for the "management" of the SC and AgentPer which handles the "disturbances". This last agent makes it possible for the model to be open and extensible in order to consider a large variety of disturbances so as to cover various types of SC (world size, national size, branch of industry, etc.). In the case of an actor of the distributor type, the agents AgentPro and AgentApp do not exist. Figure 2 
shows the implementation level of the AUML (Agent Unified Modeling Language) class diagram for the agent AgentSCM.

\begin{tabular}{|c|}
\hline $\begin{array}{c}\text { «agent » } \\
\text { AgentSCM }\end{array}$ \\
\hline $\begin{array}{l}\text { Statechart } \\
\text { AgentSCM_Behavior }\end{array}$ \\
\hline $\begin{array}{l}\text { Attribute } \\
\text { id } \\
\text { name } \\
\text { implemented SCM Methods } \\
\text { partnerSuppliersList } \\
\text { partnerCustomersList }\end{array}$ \\
\hline 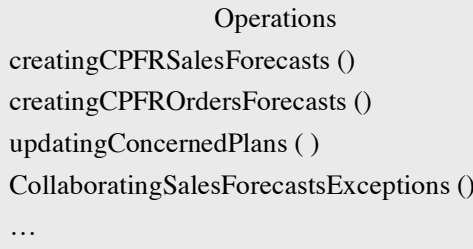 \\
\hline $\begin{array}{l}\text { Protocol } \\
\text { CPFR Protocol } \\
\text { VMI Protocol } \\
\ldots\end{array}$ \\
\hline
\end{tabular}

Fig2. Example of a class diagram (Implementation level) for the "AgentSCM"

\subsection{The safety inventory case study}

To illustrate this methodology we use an industrial example based on the rush unexpected orders and the safety inventory management. Safety inventory is necessary to compensate the uncertainty that exists in a supply chain. Retailers and distributors do not want to run out of inventory in the face of unexpected customer demand or unexpected delay in receiving replenishment orders so they keep safety stock on hand. As a rule, the higher the level of uncertainty is, the higher the level of safety stock is required. 
Safety inventory for an item can be defined as the amount of inventory on hand for an item when the next replenishment EOQ (the Economic Order Quantity) lot arrives. This means that the safety stock is an inventory that does not turn over. Basically, it becomes a fixed asset and it drives up the cost of carrying inventory. Companies need to find a balance between their desire to carry a wide range of products and offer high availability on all of them and their conflicting desire to keep the cost of inventory as low as possible (Hugo 2003b). That balance is reflected quite literally in the amount of safety stock that a company carries.

In practice, the safety stock is not enough to cover all types of unexpected swings in demand and the unexpected exceptions. As an example, the case of a customer (retailer, wholesalers, etc) who contacts his supplier (distributor) and asks for a product quantity as an immediate request, and the supplier discovers that his safety stock is lower than the ordered quantity at that moment. If this happens after the item has been logged in as a confirmed order, will the supplier be able to respond within a suitable timeframe to the customer?

We propose hereafter an agent-based distributed architecture to solve this problem of rush unexpected orders. The rush unexpected order is an object which is characterized by two attributes, the ordered quantity and the short delivery time. Multiple causes of rush unexpected order exist, they closely depend on the branch of supply chain sector. For distributors, the main interest got from such rush unexpected order delivery depends on the customer's profile and other additional criteria such as:

- It allows interesting benefit;

The distributor does not look for benefit, but he only seeks to make the customer faithful. It is the case for a wholesaler strategic customer for example.

- $\quad$ It helps increasing the number of customers. It is the case of a wholesaler who is a customer of another distributor or a regular customer who is in hurry, which will appreciate the offered service and may become a new customer.

- $\quad$ It helps a wholesaler to get rid of his surplus inventory

\section{The multi-agent architecture}

\subsection{The modeling principle}

A distribution system needs to include one or more customers who are defined by having a demand on a given product. In addition, the system needs to include one or more sources, which are defined by producing or containing the product demanded by the customer(s). Finally, the system needs a connection between the source(s) and customer(s), which can accommodate a flow of the product from the source(s) to the customer(s) in order to obtain fulfillment of the demand.

Let us suppose that a wholesaler or a particular customer had placed a rush unexpected order which is characterized by two attributes: 
- The ordered quantity OQ which cannot be delivered partially or completely from the distributor's available inventory;

- The delivery time DT.

Two cases are then possible:

a. The distributor does not have any part of the ordered quantity OQ;

b. It has a part of the ordered quantity and must complete the rest.

In both situations:

$\mathrm{OQ}=\mathrm{Dis}+\mathrm{RQ}$

Where DisQ: available quantity which can be delivered by the distributor. Two situations are possible: $\mathrm{Dis} Q=0$ (a) or DisQ $<$ OQ (b).

RQ: required quantity which must be looked for.

The problem can be then summarized as follows: find the required quantity RQ in order to deliver the rush unexpected order while respecting the delivery time DT.

One of the practices of the distributors consists of seeking the required quantity from another wholesaler or distributor. In general, the distributor will be limited to some close and faithful wholesalers or to distributors of the same company. This shows that the chosen solution (if it is found) can not be the best. Moreover, it takes enough time since the negotiation is carried out generally by phone. Within the context of SCM, we propose to extend this practice by involving (into collaboration) several wholesalers, same products distributors and equivalent products distributors. So, the distributor will be supplied from three different actors:

-1 1st type: (distributor and its wholesalers)

- Wholesalers belonging to the same area as the customer;

- Wholesalers belonging to different areas from the customer;

- Both cases above.

- 2nd type: (distributor/same products distributors)

- The same products distributors and/or their wholesalers.

-3rd type: (distributor/equivalent products distributors)

- The equivalent product distributors and/or their wholesalers.

-4th type:

- Hybrid Solution: more than the two preceding cases.

In practice, to implement this process in the industrial cases, and in order to satisfy the distributors' needs, three conditions must be checked:

- Quick and automatic solution. The manager contacts the collaborative participants only to confirm the solution.

- Autonomy and data confidentiality of each participant must be guaranteed since they can be independent.

- Transportation costs should be minimized, which will be added to the basic high purchase price since the products will not be delivered directly by the distributor.

As shown in the background review (see Sect. 2), the agent technology is more suitable for this type of problem. In this respect, we propose in the section below an agent-based distributed architecture which implements these processes. 


\subsection{The multi-agent architecture}

The proposed system does not substitute the existing tools and the SCM strategies and practices, but it can be used as a complement which improves them in the case of the presence of a rush unexpected order. The system architecture is shown in Figure 3.

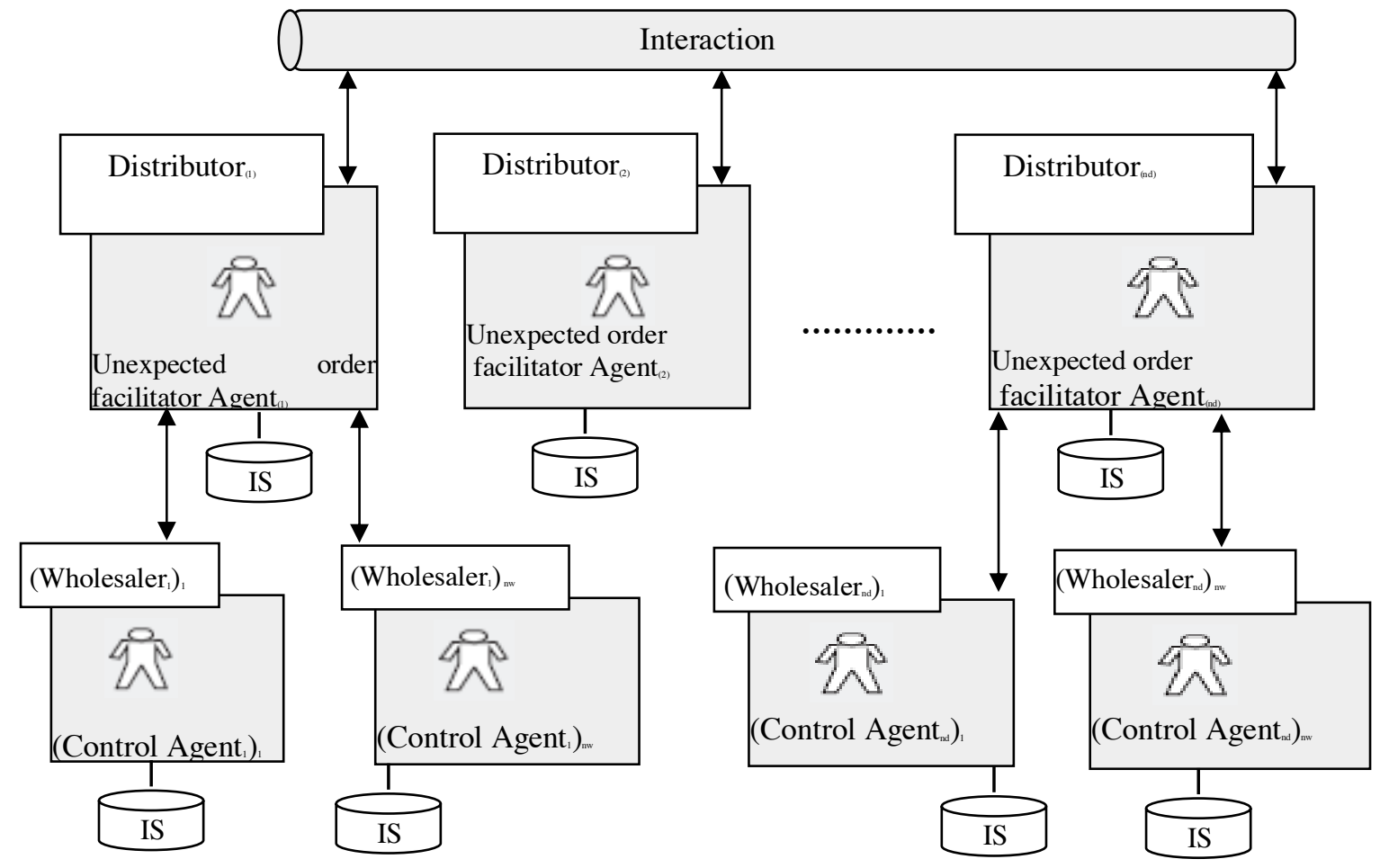

nd: the number of collaborative distributors

$\mathbf{n w}_{1}$ : the total number of collaborative wholesalers of the distributor

$\mathbf{n w}_{\mathrm{na}}$ : the total number of collaborative wholesalers of the distributor ${ }_{\text {axi }}$

IS: Information System (such as an ERP Enterprise Resources Planning system)

Fig3. Multi-Agent system architecture

The main purpose of this system is to coordinate all collaborative participants in order to find the OQ quantity and minimize total cost of transportation when a rush unexpected order is presented. The complete architecture includes two types of agents: control agents "WhoAgent" for wholesalers and the unexpected order facilitator agents "DisAgent" for distributors. Each distributor is modeled by a 


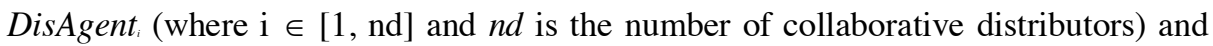
each wholesaler is modeled by a (WhoAgent), (where $\mathrm{j} \in\left[1, \mathrm{nw}_{\mathrm{i}}\right]$ and $n w_{i}$ is the total number of collaborative wholesalers of the distributor ${ }_{\varpi}$ ). These coordinated agents have the ability to specify both static and dynamic characteristics of various supply chain entities (Lee 1997); in particular, the level of distributors and their wholesalers.

The control agent plays a liaison role between a supply chain manager and the system. It collects strategies from managers, seeks the accurate data and aims at building a rule-base for better coordination and better decision-making process. When an unexpected order facilitator agent DisAgent asks a control agent (WhoAgent), (where $\mathrm{j} \in\left[1, \mathrm{nw}_{\mathrm{w}}\right]$ and $\mathrm{nw}_{\mathrm{i}}$ is the total number of collaborative wholesalers of the distributor $\left._{\omega}\right)$ for possible quantity of product that it can deliver, control agent (WhoAgent.), sends information about possible quantity and costs, and transportation costs. The agent system is autonomous because it allows any manager to change strategies of that node. Also, it allows overcoming the hurdle which consists of need for sharing sensitive information of participants in the SC, because the agents do not exchange information about inventory levels and strategies. In real world coordination, sharing information truthfully is problematic since intra-organizational trust cannot be easily developed. Next, if the collaborative participants (wholesalers and their distributors) are independent and operate within the same sector (same or equivalent products); information sharing becomes a critical obstacle, since each independent wholesaler/distributor is typically not willing to share with the other nodes its own strategic data (as inventory level for example). On the real global distribution cases, and especially in the same distribution sector, it is easy to implement the interaction between a distributor and its wholesalers. However, it is difficult, even impossible, to implement it between a distributor and the wholesalers of another distributor. Indeed, the participants are confronted to the increasing impact of competitive pressures. Also, each distributor tries to increase the number of its customers. To represent the real global distribution cases, each agent DisAgent can interact with other agents $\left\{\right.$ DisAgent $\left._{*} / k \in[1, n d], k \neq i\right\}$ and control agents $\left\{(\text { WhoAgent })_{1}, / j \in\left[1, n w_{i}\right]\right\}$, but a control agent can interact only with its distributor agents.

The unexpected order facilitator agent DisAgent, which communicates with control agents and other unexpected order facilitator agents, plays the same role of control agent. In addition, it provides the best solution of solving the rush unexpected order, which tries to minimize the total transportation costs. When a rush unexpected order (which characterized by: OQ and DT) is presented at the distributor ${ }_{\omega}$, the manager asks the unexpected order facilitator agent DisAgent to search the best solutions. At this moment, the agent carries out the algorithm "search_OQ" for solving the problem which can be summarized as follows:

- Search the RQ: it can be delivered completely by only one wholesaler or gathered from several wholesalers (1st type). 
- Find and classify a series of possible paths in an ascending order depending on transportation cost (which depends closely on the distance) to transport OQ while respecting the delivery time DT.

Propose quantity $\mathrm{Q}$ to be supplied by each participant belonging to a path.

Figure 4 shows the UML (Unified Modeling Language) sequence diagram (Bauer and Odell, 2005) that expresses the exchange of messages through the interaction protocol between the unexpected order facilitator agent DisAgent and the control agents $\left\{(\right.$ WhoAgent $\left.), / j \in\left[1, n w_{i}\right]\right\}$.

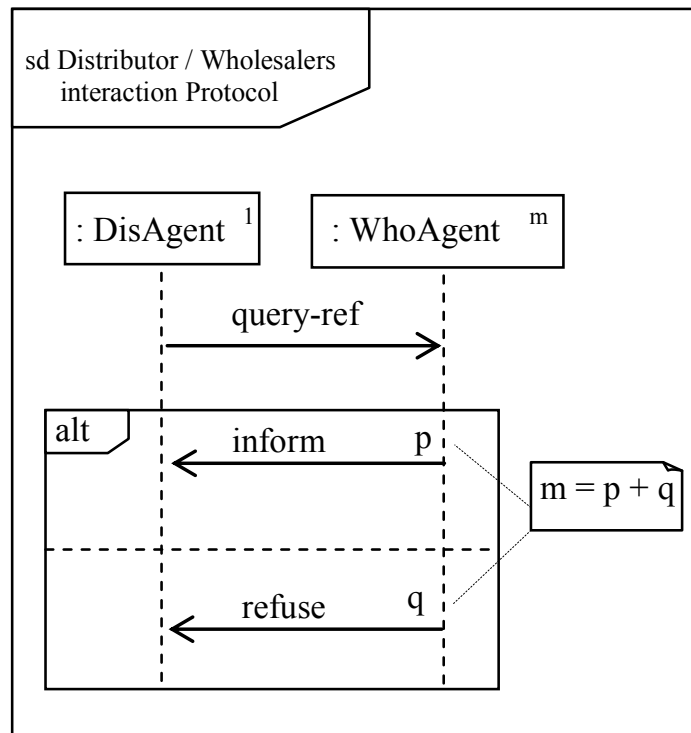

Fig4. Distributor / Wholesalers interaction protocol

If no solutions are found or the manager is not satisfied by the proposed solutions, the agent can interact with the unexpected order facilitator agents $\{$ DisAgent $/ k \in[1$, $n d], k \neq i\}$ of other distributors in order to find the best solution. In this case, the agent DisAgent sends a message of performative QUERY-REF to all the collaborative unexpected order facilitator agents to find the $R Q$. Each agent applies his decisionmaking process in order to deliver the $R Q$. They can interact if necessary with the control agents to get the best solution. At the end, the unexpected order facilitator agent DisAgent sorts all the received solutions. Figure 5 shows the UML sequence diagram that expresses the exchange of messages through the interaction protocol between the unexpected order facilitator agents of several distributors. 


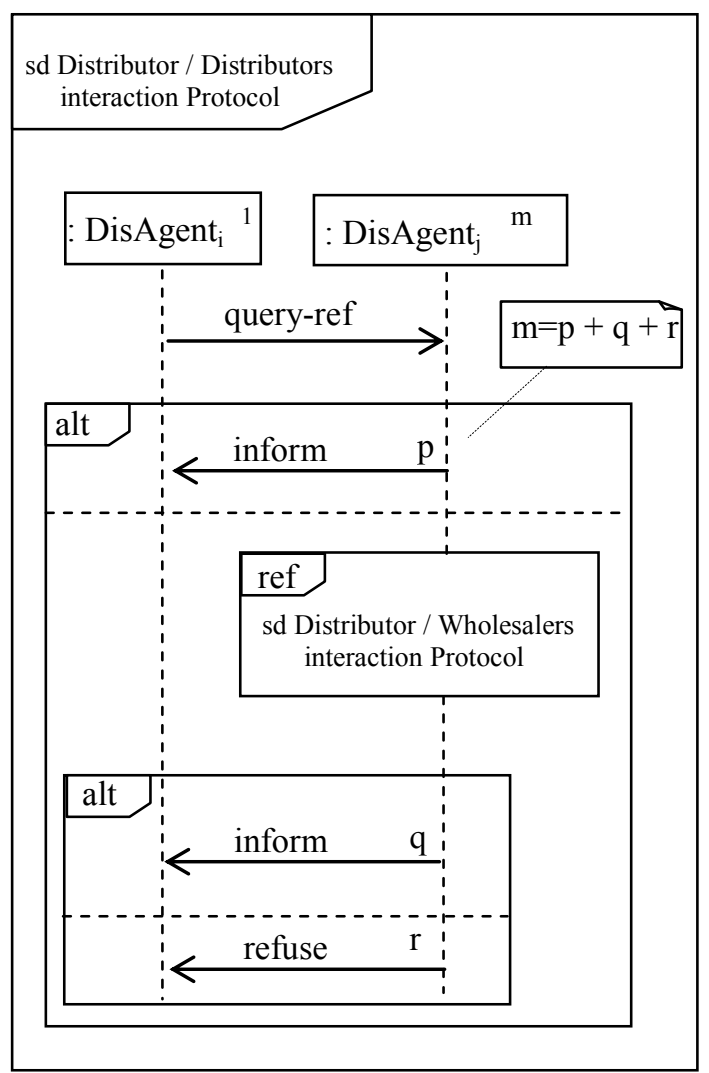

Fig5. Distributor / Distributors interaction protocol

\subsection{Negotiation protocols}

The negotiation is the mechanism by which the agents can establish a common agreement. In the case of intelligent agents and of the MAS (Multi-Agent Systems), the negotiation is a basic component of the interaction because the agents are autonomous (Jenning et al. 2001); there is no solution imposed in advance and the agents must find solutions dynamically, while solving the problems. To model the negotiation between the agents composing our system, we consider the following aspects:

- The negotiation object: an abstract object which includes the attributes that the agents want to negotiate. In our architecture, several objects are prone to be negotiated according to the situation. We find among others, the Order and its attributes (quantity and delivery date), the Contract of Continuous Delivery and its attributes (quantities and plan of delivery), the Forecasts and their attributes 
(quantities, dates and exceptions) and the acceptable scenario in the case of dysfunctions.

- The decision-making process: this is the model that the agent uses to make the decisions during the negotiation. The most important part of making decisions is the negotiation strategy which allows the agent to choose the most appropriate communicative intention (also called "performative") at a certain time. The performative can be ACCEPT_PROPOSAL, REQUEST, INFORM, PROPOSE, etc.

- The communication language: the language used by the agents to exchange their knowledge and information during the negotiation. We use the FIPA-ACL language (FIPA 2002) in our application.

- The negotiation protocol: the set of elements that governs the negotiation such as the possible participants in the negotiation, the legal proposals that the participants can make, the states of the negotiation, and finally, a rule to determine when the negotiation should be stopped in case of agreement (or when it is necessary to stop the negotiation process because no agreement could be reached).

In the SCM process, the agents are co-operative, having the same goal (aggregation of the local objectives). They share and solve problems together. For this reason, the agents must provide useful reactions to the proposals that they receive. These reactions can take the form of a counter-proposal (refused or modified proposal). A counter-proposal is an alternative proposal generated in response to a proposal. From such reactions, the agent must be able to generate a proposal which is probably ready to lead to an agreement. Consequently, the agents of our system must use protocols respecting the criteria which have been stated above and that mainly depend on three parameters:

- The branch of supply chain sector (textile and clothing sector, consuming goods sector, etc.);

- SCM strategies and practices used for the companies' co-operation and coordination;

- Objects to be negotiated: rush order, ordinary order, sales forecasts, orders forecasts; modification of delivery plans in case of trouble, etc.

\section{Heuristic negotiation}

Figure 6 shows, as an example, the heuristic negotiation (Florea 2002). In this protocol several proposals and counter-proposals can be exchanged in various steps. Agent "A", with proposal "pA", is the initiator of the negotiation, whereas the agent "B" (participant) can reply with the answers "p1B", "p2B" and "p3B" (to modify the request).

The number of the counter-proposals is limited. Once this limit is reached, the agents arrive to a rejection. We propose to recapitulate the heuristic negotiation protocol using UML sequence diagram (figure 6). 


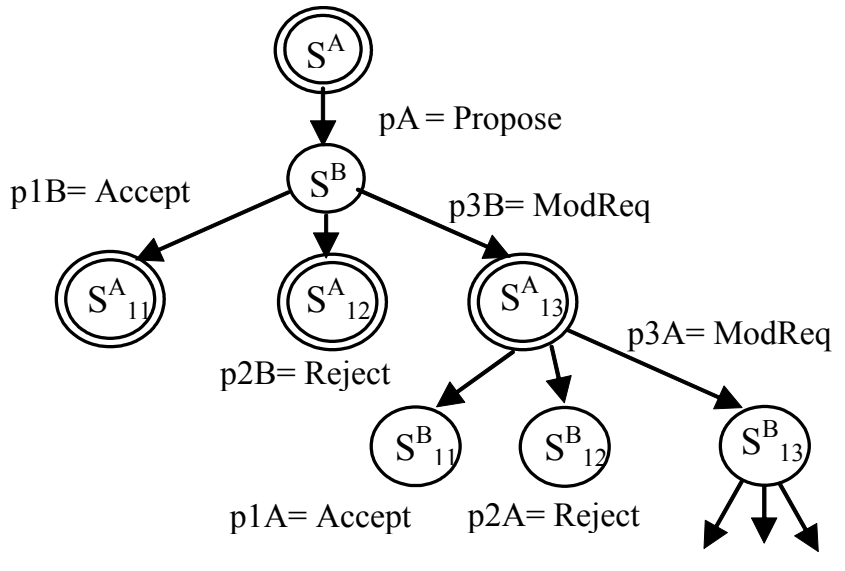

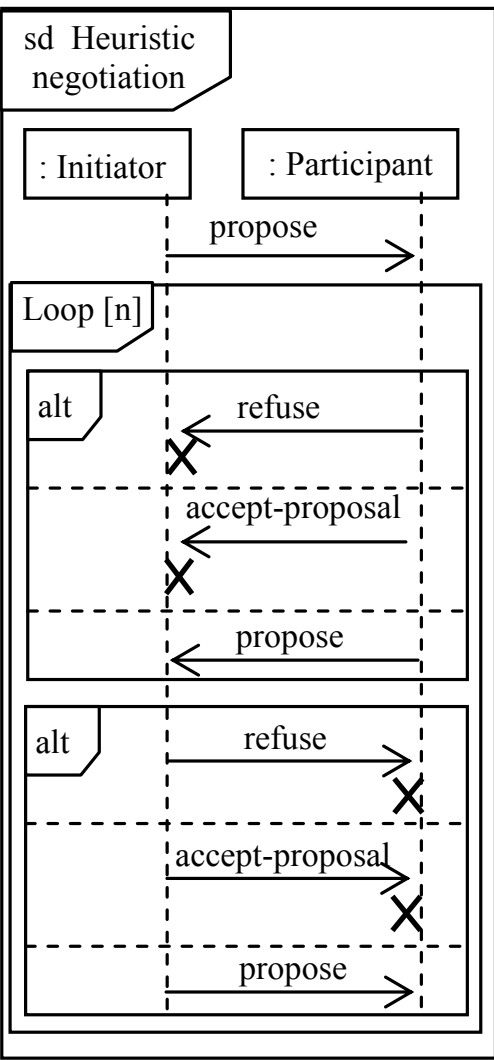

Fig6. Example of a heuristic negotiation protocol

\section{Industrial case study}

A case study of the proposed agent-based distributed architecture is conducted at a leading distribution company in North Africa. The aforementioned company operates in the sectors of toilets and showers (washbasin, baths, etc.), taps, tiling, plumbing and pieces of furniture. It offers about 800 economical and high-end items at both its wholesale and retail market locations which are geographically distributed throughout the region. The products are sourced from two national suppliers (located in Morocco) and two main foreign suppliers (located in Spain and Turkey). The distributor mainly manages three types of independent-demand inventory systems: Periodic Review system (sometimes called a fixed interval reorder system or periodic reorder system) which is used to manage the inventory of the high-end products sourced from the foreign suppliers; a new order is always placed at the end of each review; the Time Between Orders (TBO) is fixed at 6 months for high-end items of plumbing, and 1 month for high-end items of tiling. The reorder point system (or fixed ordering quantity system), which tracks the remaining inventory of an item (sourced from national suppliers) each time a withdrawal, is made to determine whether it is time to reorder. Demands are estimated from forecasts and/or customer orders. A sourcing-to- 
order is used for some items depending closely on taste and last fashion (like products of tiling, etc).

The distributor has noticed that, in the whole, there are 6 to 10 rush unexpected orders that could take place each month. Actually, it is a big challenge because the aforementioned orders do not concern the same product. At the average level, $60 \%$ of such orders are cancelled, $20 \%$ are made by other distributors, and the delivery time of the other $20 \%$ can be modified after a tough negotiation with the customer.

At the beginning of the year (the beginning of the low sales season), a real-estate operator (who already promised the delivery of the apartments to the customers at a precise time) must have closed the building site (located in Fez city in the central part of Morocco) during one week in order to have the housing license agreement as soon as a high-end item required in the construction had been in shortage. This latter is due to the inventory shortage of this item at the distributor. Because of a problem in the supplying process mainly due to one of the foreign suppliers, the next planned delivery of this item has been backlogged. In addition, the required high-end item cannot be supplied from other distributor because the aforementioned distributor is the only exclusive actor of such product. This order placed during the first week of the month was regarded as an unexpected firm rush order (delivery time: 9 hours; and it can neither delayed nor cancelled).

\section{a. Adopted solution}

In this emergency case, the distributor was compelled to place an order of an equivalent item (mark, color...) at another competitor distributor located in Casablanca city (about $289 \mathrm{Km}$ from Fez city). Even if the costs were high, the distributor was interested in rendering the real-estate operator faithful.

b. Solutions provided by the proposed system

In order to show the effectiveness and usefulness of the suggested architecture, we have made use (as a tangible demonstration) of the aforementioned item inventory level history of 37 wholesalers distributed throughout the country. They are identified by $\mathrm{scm} 1, \mathrm{scm} 2 \ldots \mathrm{scm} 37$.

13 existing solutions (paths and quantities) have been proposed by the unexpected order facilitator agent, according to the value of the number of wholesalers involved in each path. This number is chosen by the manager; the Qmin is the minimal quantity of product which can be transported in a segment connecting a wholesaler and the next wholesaler in the same path.

Results comment:

- We notice that even if the required quantity cannot be delivered completely by only one wholesaler, it can gathered from both wholesalers scm26 (Ourzazate) and $\mathrm{scm} 23$ (Marrakech). This solution is not appropriate since the distance of the path is $687 \mathrm{~km}$. Indeed, the costs of transportation will be high.

- The distributor has 12 choices concerning the paths including 3 wholesalers. The time of the first three paths are almost equal to half of the required delivery time. The 
distances $(323 \mathrm{~km}, 343 \mathrm{~km})$ of the first two paths (Tetouan-Chefchaouen-Meknes-Fez; Tanger-Tetouan-Chefchaouen-Fez) are about $289 \mathrm{~km}$ (distance between the competitor distributor and the customer). Certainly, these two solutions are better than the adopted solution, at least for two reasons:

1. The costs of transportation will be almost the same;

2. The purchase prices offered by the collaborative wholesalers (involved in the same supply chain) are lower than the offered prices from the competitor distributor.

We deduce that the distributor had three solutions better than the adopted one. We can conclude that more the number of actors constituting a path and the required quantity are large, more the number of choices are important. In practice, several emergency situations exist and depend closely on the branch of supply chain sector.

\section{Conclusion and perspectives}

This chapter was dedicated to an agent-based distributed architecture for collaborative decision-making processes within the global distribution supply chain.

A process to better manage the rush unexpected orders, thanks to the multi-agent properties, has been presented. A generic model, allowing a flexible modeling of the supply chain is used. This model makes it possible to test a set of negotiation protocols that have been previously proposed and modeled with the UML language. The used scenario shows that UML diagrams offer effective solutions to specify and model real-world agent-based applications. At this stage, the architecture was validated and tested on a leading distribution company. The results are very promising and show that the system could be connected to the existing $\mathrm{SC}$ tools.

An enhancing stage could consider the size of the transported batches, i.e. to represent the price of transportation per unit of product as being lower when the transported quantity is large. In this case, it is necessary to seek the quantities which optimize the costs of transportation (using for example genetic algorithms). Then, the presented architecture could be enhanced to be used in a global manufacturing supply chain (including manufacturers, suppliers...).

\section{References}

Arntzen BC, Brown GG., Harrison TP. and Trafton LL (1995) Global supply chain management at digital equipment corporation. Interfaces 25(1): 69-93.

Bagchi S, Buckley S, Ettl M, and Lin G (1998) Experience using the IBM supply chain simulator. In Proceedings of the 1998 Winter Simulation Conference: 1387-1394, Washington, D.C, USA. 
Baglin G, Bruel O, Garreau A, Greif M, and Delft Ch (2001) Management Industriel et Logistique. Collection Gestion, Série: Productique et techniques quantitatives appliquées à la gestion, Economica, 323-340.

Banks J, Jain S, Buckley S, Lendermann P and Manivannan M (2002) Panel Session: Opportunities for Simulation in Supply Chain Management. In Proceedings of the 2002 Winter Simulation Conference: 1652-1658. San Diego, California.

Bauer B, \& Odell J (2005) UML 2.0 and agents: How to build agent-based systems with the new UML standard. Engineering Applications of Artificial Intelligence, 18, 141-157.

Brun A, Cavalieri S, Macchi M, Portioli-Staudacher A and Terzi S (2002). Distributed simulation for supply chain co-ordination. In Proceedings of the 12th International Working Seminar on Production Economics, Igls, Austria.

Calvin B, Lee Ph.D. (2003) Multi-Echelon Inventory Optimization. Evant White Paper Series.

Chehbi S, Derrouiche R, Ouzrout Y and Bouras A (2003) Multi-Agent Supply Chain Architecture to Optimize Distributed Decision Making. Proceedings of the 7th World Multi-conference on Systemics, Cybernetics and informatics, SCI, 16, Orlando, USA,.

Chen F, Drezner Z, Ryan J.K and Simchi-Levi D (2000a) Quantifying the bullwhip effect in a simple supply chain: the impact of forecasting, lead times, and information. Management Science, 46(3): 436-443.

Chen F, Drezner Z, Ryan J.K and Simchi-Levi D (2000b) The impact of exponential smoothing forecasts on the bullwhip effect. Naval Research Logistics, 47: 269- 286.

Cloutier L, Frayret J-M, D'Amours S, Espinasse B and Montreuil B (2001) A commitmentoriented framework for networked manufacturing coordination. International journal of computer integrated manufacturing, 14(6): 522-534.

Cohen MA, Lee HL (1988) Strategic analysis of integrated production distributed systems: models and methods. Oper Res, 36(2): 216-228.

ECR (2006). http://www.ecrnet.org/. (Last access: January 12, 2006).

FIPA (2002) FIPA ACL Message Structure Specification. Foundation for Intelligent Physical Agents. (On http://www.fipa.org/spcs/fipa00061/SC00061G.pdf).

Florea A (2002) Using Utility Values in Argument-based Negotiation. In Proceedings of ICAI'02, the 2002 International Conference on Artificial Intelligence: 1021-1026, Las Vegas, Nevada, USA.

Fu Y, Souza R.D and Wu J (2000) Multi-agent enabled modeling and simulation towards collaborative inventory management in supply chain, Proceedings of the 2000 Winter Simulation Conference, 2: 1763-1771, Wyndham Palace Resort \& Spa, Orlando, FL, USA.

Fujimoto R (1999) Parallel and distributed simulation. In Proceedings of the 1999 Winter Simulation Conference: 122-131, IEEE, Piscataway, NJ.

Giannoccaro I, Pontrandolfo P, and Scozzi B (2003) A fuzzy echelon approach for inventory management in supply chains, European Journal of Operational Research, 149(1): 185196.

Hieber R (2002) Supply chain management: a collaborative performance measurement approach. VDF (eds.).

Hugos M (2003a) Essentials of Supply Chain Management. John Wiley \& Sons (eds.): 3-4.

Hugos M (2003b) Essentials of Supply Chain Management, John Wiley \& Sons (eds.): 64-65.

Jennings N. R, e.a. (2001) Automated negotiation: Prospects, methods and challenges. Group Decision and Negotiation Journal, 10(2): 199-215.

John Taras CPIM (2007) Vmi web site. (Last access: April 15, 2007).

Kimbrough S.O, Wu D.J and Zhong F (2002) Computers play the beer game: can artificial agents manage supply chains?. Decision Support Systems, 33(3): 323-333. 
Lee H.L, Padmanabhan V, and Whang S (1997) The bullwhip effect in supply chains. Sloan Management Review, 38(3): 93-102.

Maturana F, Shen W and Norrie DH (1999) MetaMorph: an adaptive agent-based architecture for intelligent manufacturing. International Journal of Production Research, 37(10): 21592174.

McBurney P, Parsons S, Green J (2002) Forecasting market demand for new telecommunications services: an introduction. Telematics and Informatics, 19(3): 225249.

Moyaux T, Chaib-draa B, and D'Amours S (2004) An agent simulation model for the québec forest supply chain, Proceedings of the 8th International Workshop on Cooperative Information Agents (CIA), 3191: 226-241, Erfurt, Germany.

Nfaoui E.H, Ouzrout Y, El Beqqali O and Bouras A (2006) An approach of agent-based distributed simulation for supply chains: Negotiation protocols between collaborative agents. Proceedings of the 20th annual European Simulation and Modelling Conference: 290-295. EUROSIS Publication ISBN: 90-77381-30-9. Toulouse, France,

Parunak H. V. D. 1996 Applications of distributed artificial intelligence in industry. In O'Hare, G. M. P. And Jennings, N. R., editors, Foundations of Distributed Artificial Intelligence: 71-76, John Wiley et Sons (eds.).

Petersen S.A, Divitini M and Matsken M (2001) An agent-based approach to modeling virtual enterprises. Prod Plan Control 2001; 12(3): 224-233.

Pinedo M and Chao X (1999) Operations Scheduling with applications in manufacturing and services. Mc. Graw-Hill (eds.).

Reutterer T, Kotzab H.W, (2000) The use of conjoint-analysis for measuring preferences in supply chain design. Industrial Marketing Management, 29(1): 27-35.

Sadeh NM, Hildum DW, Kjenstad D and Tseng A (2001) MASCOT: an agent-based architecture for dynamic supply chain creation and coordination in the internet economy, Prod Plan Control, 12(3): 212-223.

Samii A-K (2004) Stratégie Logistique, Supply Chain Management. Dunod (eds.), $3^{\text {st }}$ edition: 14-15.

Siala M, Campagne J and Ghedira K (2006) Proposition d'une Nouvelle Approche pour la Gestion du Disponible dans les Chaînes logistiques. 6e Conférence Francophone de MOdélisation et SIMulation (MOSIM): 412-421, 3-5 Apr, Rabat, Maroc.

Simchi-Levi D, Kaminsky P and Simchi-Levi E (2000) Designing and managing the supply chain, McGraw-Hill Higher Education: 239.

Swaminathan J.M, Smith S.F and Sadeh N.M (1998) Modeling supply chain dynamics: a multiagent approach, Decision Sciences, 29(3): 607-632.

Telle O, Thierry Caroline and Bel G (2003) Simulation d'une Relation Client/Fournisseur au sein d'une Chaine Logistique Intégrée: Mise en Oeuvre Industrielle. In Proceedings of MOSIM03 (Conférence Francophone de MOdélisation et Simulation), Toulouse, France.

Terzi S, Cavalieri S (2004) Simulation in the supply chain context: a survey. Computers in Industry, 53(1): 3-16.

Zhao X, Xie J and Leung J (2002) The impact of forecasting model selection on the value of information sharing in a supply chain. European Journal of Operational Research, 142(2): 321-344.

VICS Association (2007), Web site. (Last access: April 15, 2007).

Wu D.J (2001) Software agents for knowledge management: coordination in multi-agent supply chains and auctions. Elsevier Science Ltd, Expert Systems with Applications, 20(1): 51-64. 